Sādhanā Vol. 38, Part 3, June 2013, pp. 421-428. (c) Indian Academy of Sciences

\title{
Recent development of cellular manufacturing systems
}

\author{
P K ARORA ${ }^{1, *}$, A HALEEM ${ }^{2}$ and M K SINGH ${ }^{3}$ \\ ${ }^{1}$ Department of Mechanical Engineering, H R Institute of Technology, \\ Ghaziabad 201003, India \\ ${ }^{2}$ Faculty of Engineering and Technology, Jamia Milia Islamia, New Delhi 110025, \\ India \\ ${ }^{3}$ Nikhil Institute of Technology and Management, Mathura 281122, India \\ e-mail: pawanar@rediffmail.com
}

MS received 8 September 2011; revised 30 October 2012; accepted 10 April 2013

\begin{abstract}
Cellular manufacturing system has been proved a vital approach for batch and job shop production systems. Group technology has been an essential tool for developing a cellular manufacturing system. The paper aims to discuss various cell formation techniques and highlights the significant research work done in past over the years and attempts to points out the gap in research.
\end{abstract}

Keywords. Cellular manufacturing system; part families; group technology.

\section{Introduction}

Cellular Manufacturing (CM) has been emerged as a strong approach for improving operations in batch and job shop environments. In cellular manufacturing, Group Technology is used to form part families based on similar processing requirements. Parts and machines are then grouped together based on sequential or simultaneous techniques. This approach results in cells where machines are located in relative proximity based on processing requirements rather than similar functional aspects. Decision-making and accountability are more locally focused, often resulting in quality and productivity improvements.

\section{Design of cellular manufacturing system}

The problem of cell design is a very complex exercise with wide ranging implications for any organization (Wemmerlov \& Hyer 1986). Normally, cell design is understood as the problem of identifying a set of part types that are suitable for manufacture on a group of machines. However, there are a number of other strategic level issues such as level of machine flexibility, cell layout, type of material handling equipment, types and number of tools and fixture etc that should be considered as a part of cell design problem (Wemmerlov \& Hyer 1987).

*For correspondence 
In general, descriptive procedures can be classified into three major classes. The first class, which is referred to as part family identification (PFI), begins the cell formation process by identifying the families of parts first and then allocates machines to the families. The second class, which is referred to as machine groups identification (MGI). The third class of the descriptive procedures, which is referred to as part families/machine grouping (PF/MG), identifies the part families and machine groups simultaneously. PFI methods can be sub-classified as those based on informal systems (e.g., rules of thumb, visual examination or other criteria) and those based on formal coding and classification systems. The role of group technology (GT) codes in the context of cellular manufacturing is primarily as an aid in identifying the part families to which production cells should be dedicated. Further analysis is required before a family of parts to be manufactured in a cell, and the machines, which will comprise that cell, can be specified. MGI procedures consider the CF problem as a two-stage process where in the first stage of their analysis, machines are grouped based on information available in part routings and then in the second stage, parts are allocated to machine groups. When a CF approach attempts to group parts into part families and machines into machine groups simultaneously, then such an approach can be classified as PF/MG. Burbidge (1975) proposed one of the earliest PF/MG descriptive approaches for the CF problem which is referred to as Production Flow Analysis (PFA). PFA is a technique, which analyses the information given in route cards to form cells. A manual method for CF called 'Nuclear Synthesis' is proposed where manufacturing cells are created around 'key machines'. E1-Essawy proposed a method called Component Flow Analysis (CFA) at about the same time. In some respects, the methodology of CFA does differ from that of Burbidge's PFA procedure since the latter first partitions the problem, whereas the former does not.

\subsection{Machine component group analysis}

Logendrana et al (2003) addressed the two-machine group-scheduling problem for minimizing the mean flow time with carryover sequence dependency. Chan et al (2003) proposed algorithm uses an adoptive approach to relate machines in a cell by examining the merged part flow weights of machine pairs. The establishment of the part flow weight includes practical constrains, such as the part-handling factor and the number of parts per transportation. It should be noted that (Chan Felix et al 2006) if the production volume of each part is very large, then the total number of intercellular movement will be further larger. Therefore, the sequence of machine cells is particularly important in this aspect. Das et al (2007) presented a multi-objective mixed integer programming model of cellular manufacturing system design which minimizes the total system costs and maximizes the machine reliabilities along the selected processing routes. A part may be processed under different process plans, each prescribing a sequence of operations to be performed at various machines in serial configuration.

A multi-objective dynamic cell formation was presented by Bajestani et al (2009), where the total cell load variation and sum of the miscellaneous costs (machine cost, inter-cell material handling cost, and machine relocation cost) are to be minimized simultaneously. Since this type of problem is NP-hard, a new multi-objective scatter search (MOSS) is designed for finding locally Pareto-optimal frontier. Taboun et al (1998) implemented a two-stage procedure for cost effective part family and machine cell formation. First, the problem is formulated as a mixed integer mathematical model for simultaneous machine grouping and part family assignment. Sudhakara \& Mahapatra (2009) presented a model in which the machines are allocated into machine cells to produce the identified part families so that productivity and flexibility of the system can be improved. Zero-one part-machine incidence matrix (PMIM) generated from route sheet information is commonly presented as input for clustering of parts and machines. An entry 
of ' 1 ' in PMIM indicates that the part is visiting the machine and zero otherwise. Noktehdan et al (2010) proposed a grouping version of differential evolution (GDE) algorithm and its hybridized version with a local search algorithm (HGDE) to solve benchmarked instances of cell formation problem posing as a grouping problem.

\subsection{Similarity coefficient based approaches}

A number of researchers or investigators have used different types of similarity and dissimilarity coefficients for determining part families. In cellular manufacturing systems, most machines are capable of performing more than one operation, which makes parts rerouting feasible. When a part is rerouted, it affects the cell performance. Most of the suggested approaches in the literature develop a new similarity coefficient based on mathematical analysis; however, these methods tend to disregard alternative routes during machine failure. Mcauley (1972) was the first to apply the Jaccard similarity coefficient to the cell formation problem. Kusiak (1987) considered alternate process plans and developed a p-median model to form cells. Choobineh (1984) proposed a new similarity measure which uses the manufacturing operations and their sequences. Gunasingh \& Lashkari (1991) suggested a new similarity index which expressed the capability between two machines in processing a set of parts that need both machines. Tam (1990) also proposed a new similarity coefficient based on the similarity of operation sequences. Seifoddini (1990) suggested a new similarity coefficient which was based on the idea that necessary production data should be incorporated in the early stages of the machine-component grouping process. They considered processing requirements of parts, pair-wise average production volume, and unit operation time as new production parameters. Gupta (1993) suggested a new similarity coefficient which required that alternative routing of parts should be considered while calculating the pair-wise similarity coefficient between machines. Kamrani \& Parsaei (1993) proposed a weighted dissimilarity coefficient based on e disagreement measure of both design and manufacturing attributes between two parts. Moussa \& Kamel (1995) also proposed a new similarity coefficient which took into consideration the operation sequences and process times during the assignment process. Garbie et al (2004) proposed new similarity coefficient which considers the number of alternative routes available during machine failure. Based on the new similarity coefficient, the part families were identified by using a p-median model. Two characteristics, discriminability and stability of the similarity coefficients are tested under different data conditions by Yin \& Yasuda (2006). From the results, three similarity coefficients are found to be more discriminable. Jaccard is found to be the most stable similarity coefficient. Yin \& Yasuda (2005) gave a comprehensive overview and discussion for similarity coefficients developed to date for use in solving the cell formation (CF) problem. Jeon et al (1998) developed a methodology which can be used to form manufacturing cells using both a new similarity coefficient based on the number of alternative routes during machine failure and demand changes for multiple periods.

\subsection{Genetic algorithm-based approaches}

Genetic Algorithm is a popular heuristic search technique and has proved superior performance on complex optimization problem. Kuroda et al (1999) formulated the designing and controlling problems of cellular-line systems and solved them using a genetic algorithm. Liu (2007) developed a data mining algorithm for designing the conventional cellular manufacturing systems. Chen (1997) explained how a genetic algorithm can be applied to vision systems for shape analysis and quality assessment. A new algorithm which will consider both design and manufacturing attributes and operation sequences as factors, is proposed by Gungor \& Arikan (2000) to 
formulate the problem. Tariq et al (2009) developed an approach that combines a local search heuristic (LSH) with genetic algorithm (GA). In the general machine/part grouping problem, processing times, lot sizes and machine capacities are all explicitly considered by Zolfagharia \& Liang (2003). To evaluate the solution quality of this type of grouping problems, a generalized grouping efficacy index is used as the performance measure and fitness function of the genetic algorithm. The algorithm has been applied to solving several well-cited problems with randomly assigned processing times to all the operations. Mahdavi et al (2009) proposed a model, with nonlinear terms and integer variables which cannot be solved for real sized problems efficiently due to its NP-hardness. Saadettin et al (2010) presented a genetic algorithm (GA) based heuristic approach for job scheduling in virtual manufacturing cells (VMCs). In a VMC, machines are dedicated to a part as in a regular cell, but machines are not physically relocated in a contiguous area.

\subsection{Neural network based approaches}

Lozano et al (2001) investigated two sequence-based neural network approaches for cell formation and the objective function considered is the minimization of transportation costs (including both intracellular and intercellular movements). Although Enke et al (1998) adopted the ART1 network with an application in machine-part CF, there are still several drawbacks to this approach. To address these concerns Yang \& Yang (2008) proposed a modified ART1 neural learning algorithm. In this modified ART1, the vigilance parameter can be simply estimated by the data so that it is more efficient and reliable than Dagli \& Huggahalli (1995) method for selecting a vigilance value. Jerbic et al (1999) implemented a planning system which integrates the reinforcement learning method and a neural network approach with the aim to ensure autonomous robot behaviour in unpredictable working conditions using non-permutation schedules, three prominent types of met heuristics - a simulated annealing, a genetic algorithm and a tabu search - are proposed and empirically evaluated by Lin et al (2009). Rezaeian et al (2011) presented a new nonlinear programming model in a dynamic environment. Furthermore, a novel hybrid approach based on the genetic algorithm and artificial neural network is proposed to solve the presented model. From the computational analyses, the proposed algorithm is found much more efficient than the genetic algorithm and simulated annealing in generating optimal solutions.

\subsection{Mathematical programming based approaches}

Mathematical programming methods can be further classified into three major groups based on the type of formulation.

2.5a Linear programming $(L P)$ : Linear programming is a concept of expressing the interrelationship of activities of a system in terms of a set of linear constraints in non-negative variables. A program, that is, values of the variables is selected which satisfies the constraints and premises a linear objective function in these variables. LP based CF methods have been proposed by Purcheck (1975). They essentially apply the technique of combinatorial grouping and LP to the CF problem. LQP models have been proposed by Kusiak (1987), Boctor (1991) and many others. GP models have been proposed by Sankaran (1990) and Shafer \& Rogers (1991). Kioon et al (2009) presented and analysed a comprehensive model for the design of cellular manufacturing systems (CMS).The developed model is a mixed integer non-linear program. Linearization procedures are proposed to convert it into a linearized mixed integer programming formulation. Computational results are presented by solving some numerical examples, extracted from the 
existing literature, with the linearized formulation. Arkat et al (2011) presented a bi-objective mathematical model to simultaneously minimize the number of exceptional elements and the number of voids in the part machine incidence matrix.

2.5b Goal programming (GP): Goal programming is a model and associated algorithm to minimize the absolute value of deviations from a set of values called goals subjected to technological constraints.

2.5c Dynamic programming $(D P)$ : Dynamic programming determines the optimum solution to ' $\mathrm{n}$ ' variable problems by decomposing it into 'n' stages with each stage constituting a singlevariable sub problem. The computational advantage is that DP optimizes single-variable subproblems. However, because of the nature of the stage differs depending on the optimization problem, DP does not provide the computational details for optimizing each stage.

\subsection{Heuristic based approaches}

The objective of many cell formation methods is to minimize overall material flows of parts. However, most methods do not incorporate several production factors that are extremely relevant to the material flows. Yin et al (2005) incorporated these factors into a nonlinear mathematical model and proposed a heuristic methodology. Chan et al (2003) presented a heuristic algorithm that addresses problems of machine should be minimal in CM. Mahdavi et al (2007) developed a heuristic algorithm-based on flow matrix for cell formation and layout design in a simultaneous fashion using sequence data. The numerical results of the algorithm on the available problems in the literature indicate the usefulness of the algorithm with regard to performance indices. Vitanov et al (2007) presented a decision support tool that can be used by practitioners and industrialists to solve practical cell formation problems. The tool is based on a cell formation algorithm that employs a set of heuristic rules to obtain a quasi-optimal solution from both component routing information and other significant production data. Ghezavati \& Saidi-Mehrabad (2011) addressed a new version of Stochastic Mixed-Integer model to design cellular manufacturing systems (CMSs) under random parameters described by continues distributions.

\subsection{Fuzzy clustering}

Selim et al (1998) classified cell formation techniques into five groups: descriptive procedure, cluster analysis, graph partitioning, artificial intelligence, and mathematical programming. Fuzzy set theory has been shown to be capable of addressing these deficiencies. For instance, Liao (2001) addressed issues involved in using classification and coding systems under a fuzzy environment. Xu \& Wang (1989) utilized fuzzy clustering to form part families and applied fuzzy pattern recognition to assign parts to pre-formed part families based on similarity coefficients. Chu \& Hayya (1991) used fuzzy c-means (FCM) to form a pre-determined number of part families and to determine a priority for reallocating parts to different families. Also using FCM, Yang \& Ching (1996) interpreted the degree of membership of a part in a cell as the ease with which the cell can process the part and defined the complementary measure.

\subsection{Artificial intelligence approaches}

Elmaraghy \& Gu (1989) presented an approach for using domain-specific knowledge rules and a prototype feature based modelling system to automate the process of identifying parts attributes 
and assigning the parts to the most appropriate manufacturing cell. The expert assignment system is based on the geometric features of the parts, characteristics of formed manufacturing cells, parts functional characteristics and attributes, as well as domain-specific manufacturing knowledge. Kusiak developed a pattern recognition based parts grouping which is similar to the grouping in GT. The basic difference between these two approaches is in the degree of automation. Application of artificial neural networks to CF problems has been proposed by Kaparthi \& Suresh (1992).

\section{Gap in research}

There has been tremendous work done by various researchers on cell formation techniques. Majority of the published works on cellular manufacturing pay very little attention towards production planning and control activities of cellular manufacturing. Many current cellular manufacturing applications are running in a non-optimal environment and their performance could be improved by optimizing the parameters. But from the available literature most of the cell formation techniques/algorithm do not discuss the optimal size of the cell and the optimal number of cells, should be formed for a given problem. The techniques discussed above also do not investigate the effect on different performance measures if the number of cell/cell size/composition of cells varied.

\section{Conclusion}

Various techniques and methodology have been briefly studied with their salient features. The study brings the attention towards the need for designing the cellular manufacturing system for optimal performance as most of the past research work has been concentrated to the clustering of the machine and parts into cell and part families. So, acute need is to develop the models to specify the optimal number of groups and optimal production mix subject to technological and logistical constraints for optimal performance of cellular manufacturing system. There is a need to develop more efficient tools enabling manufacturing system designer to achieve optimal solution in reasonable processing time. Genetic algorithms have been proved as an efficient tool for providing optimal solutions for development of cellular manufacturing systems as evident from the recent research and hence major attention should be given for GA-based approaches.

\section{References}

Arkat J, Hosseini L and Farahan M H 2011 Minimization of exceptional elements and voids in the cell formation Problem using a multi-objective genetic algorithm. Expert Syst. Appl. 38(8): 9597-9602

Bajestani M A, Rabbani M, Rahimi-Vahed A R and Khoshkhou G B 2009 A Multi- objective scatter search for a dynamic cell formation problem. Comput. Oper. Res. 36(3): 777-794

Burbidge J L 1975 The introduction of group technology, Landon: Heinenan

Boctor F F 1991 A linear formulation of the machine-part cell formation problem. Int. J. Prod. Res. 29(2): 343-349

Chan W M, Chan C Y and Ip W H 2003 A heuristic algorithms for machine assignment in cellular layout. Comput. Ind. Eng. 44(1): 49-73

Chan Felix T S, Lau K W, Chan P L Y and Choy K L 2006 Two-stage approach for machine-part grouping and cell layout problems. Comput. Ind. Eng. 22(3): 217-238 
Chen Jen-Ming 1997 A genetic based vision system for cross -functional integration in flexible manufacturing: a tutorial and application. Int. J. Flex. Manuf. Sys. 9(97): 343-365

Choobineh F 1984 Optimum loading for GT/MRP manufacturing systems. Comput. Ind. Eng 8(3-4): 197206

Chu C H and Hayya J C 1991 A fuzzy clustering approach to manufacturing cell formation. Int. J. Prod. Res. 29(7): 1475-1487

Dagli C and Huggahalli R 1995 Machine-part family formation with the adaptive resonance theory paradigm. Int. J. Prod. Res. 33(4): 893-913

Das K, Lashkari R S and Sengupta S 2007 Reliability consideration in the design and analysis of cellular manufacturing systems. Int. J. Prod. Econ. 105(1): 243-262

Elmaraghy H A and Gu P 1989 Feature based expert parts assignment in cellular. J. Manuf. Sys. 8(2): 139-143

Enke D, Ratanapan K and Dagli C 1998 Machine-part family formation utilizing an ART1 neural network implemented on a parallel neuro-computer. Comput. Ind. Eng. 34(1): 189-205

Garbie I H, Parsaei H R, Leep H R and Wong J P 2004 A New Similarity Coefficient between a New Part and the Existing Manufacturing Cells. Proceedings of the Annual IIE Conference, 691

Ghezavati V R and Saidi-Mehrabad M 2011 An efficient hybrid self-learning method for stochastic Cellular manufacturing problem: A queuing-based analysis. Expert Syst. Appl. 38(3): 1326-1335

Gupta T 1993 Design of manufacturing cells for flexible environment considering alternative routing. Int. J. Prod. Res. 31(6): 1259-1273

Gunasingh K Raja and Lashkari R S 1991 Simultaneous grouping of parts and machines in cellular manufacturing systems-An integer programming approach. Comput. Ind. Eng 20(1): 111-117

Gungor $\mathrm{Z}$ and Arikan F 2000 Application of fuzzy decision making in part- machine grouping. Int. J. Prod. Econ 63(2): 181-193

Jeon G, R Leep H and Parsaei Hamid R 1998 A cellular manufacturing system based on new similarity coefficient which considers alternative routes during machine failure. Comput. Ind. Eng. 34(1): 21-36

Jerbic B, Katarina G and Vranjes B 1999 Autonomous agent based on reinforcement learning and adaptive shadowed network. Artificial Intelligence in Engineering. 13(2): 141-157

Kamrani Ali K and Parsaei H R 1993 A Group technology based methodology for machine cell formation in a computer integrated manufacturing environment. Comput. Ind. Eng. 24(3): 431-447

Kaparthi S and Suresh N C 1992 Machine-component cell formation in group technology: a neural network approach. Int. J. Prod. Res. 30(6): 1353-1357

Kioon S A, Bulgak A A and Bekta T 2009 Integrated cellular manufacturing systems design with production planning and dynamic system reconfiguration. Eur. J. Oper. Res. 192(2): 414-428

Kuroda M, Tomita T and Maeda Kazuhiko 1999 Dynamic control of a cellular- line production system under variations in the product mix. Int. J. Prod. Econ 60-61(1): 439-445

Kusiak A 1987 The generalized group technology concept. Int. J. Prod. Res. 25(4): 561-569

Liao Warren T 2001 Classification and coding approaches to part family formation under a Fuzzy Environment. Fuzzy Sets Syst. 122(3): 425-44

Liu G 2007 A Data mining algorithm for designing the conventional cellular manufacturing systems. Advances in artificial intelligence M A Orgun and J Thornton (eds.): 715-720

Lozano S, Canca D, Guerrero F, J M and Garcia J M 2001, Machine Grouping Using Sequence- based Similarity Coefficients and Neural Networks. Rob. Comput. Integr. Manufact. 17(5): 399-404

Lin Shih-Wei, Ying K-C and Lee Z-J 2009 Meta heuristics For Scheduling A non- permutation flow line manufacturing cell with sequence dependent family setup times. Comput. Oper. Res. 36(4): 1110-1121

Logendrana R, Gelogullaria Cumhur A and Sriskandarajah C 2003 Minimizing the mean flow time in a two-machine group-scheduling problem with carryover sequence dependency. Rob. Comput. Integr. Manufact. 19: 21-33

Mahdavi Iraj, Javadi B and Fallah-A K 2007 Designing a new mathematical model for cellular, manufacturing system based on cell utilization. Appl. Math. Comput. 190(1): 662-670

Mahdavi Iraj, Mahdi P M, Solimanpur M and Heidarzade A 2009 Genetic algorithm approach for solving a cell formation problem in Cellular manufacturing. Expert Syst. Appl. 36(3): 6598-6604 
Mcauley J 1972 Machine Grouping For Efficient Production. Production Engineers 51(2): 53-57

Moussa S E and Kamel M S 1995 Partitioning Techniques For Cellular Manufacturing. Manufact. Res. Technol. 24: 73-95

Noktehdan A, Karimi B and Kashan A H 2010 A differential evolution algorithm for the manufacturing cell formation problem using group based operators. Expert Syst. Appl. 37(7): 4822-4829

Purcheck G F K 1975 A Linear - Programming method for the combinatorial grouping of an Incomplete set. Journal of Cybernetics 5(4): 51-54

Rezaeian J, Javadian N, Tavakkoli- M R and Jolai F 2011 A hybrid approach based on the genetic algorithm and neural network to design an incremental cellular manufacturing system. Appl. Soft Comput. 11(6): 4195-4202

Sankaran S 1990 Multiple objective decision making approach to cell formation: A goal Programming model. Mathematical Computer Modeling 13(9): 71-76

Saadettin E K, Das Sanchoy K and Gungo Z 2010 A genetic algorithm-based heuristic for scheduling of virtual Manufacturing cells (VMCs). Comput. Oper. Res. 37(6): 1148-156

Selim Hassan M, Askin Ronald G and Vakharia Asoo J 1998 Cell formation in group technology: review, evaluation and directions for future research. Comput. Ind. Eng. 34(1): 3-20

Seifoddini H 1990 Machine-component group analysis versus the similarity coefficient method in cellular manufacturing applications. Comput. Ind. Eng. 18(3): 333-339

Shafer S N and Rogers D F 1991 A goal programming approach to cell formation Problems. J. Oper. Manag. 10(1): 28-33

Sudhakara Pandian R and Mahapatra S S 2009 Manufacturing Cell Formation with Production Data Using Neural Networks. Comput. Ind. Eng. 56(4): 1340-1347

Tam K Y 1990 An operation sequence based similarity coefficient for part family. J. Manuf. Syst. 9(1): $55-68$

Tariq Adnan, Hussain I and Ghafoor A 2009 A Hybrid Genetic Algorithm for Machine-Part Grouping. Comput. Ind. Eng. 56(1): 347-356

Taboun S M, Merchawi N S and Viger T 1998 Two-Stage model for cost effective part family and machine cell formation. Comput. Ind. Eng. 34(4): 759-776

Vitanov V, Tjahono B and Marghalany I 2007 A Decision support tool to facilitate the design of cellular manufacturing layouts. Comput. Ind. Eng. 52(4): 380-403

Wemmerlov U and Hyer N L 1986 Procedure for the part family machine group identification problem in cellular manufacturing. J. Oper. Manag. 6(2): 125-147

Wemmerlov, U and Hyer N L 1987 Research issue in cellular manufacturing. Int. J. Prod. Res. 25(3): $413-43$

Xu H and Wang H P 1989 Part family formation for GT applications based on fuzzy mathematics. Int. J. Prod. Res. 27(9): 1637-1651

Yang W H and Ching J L 1996 Group scheduling on two cells with inter cell movement. Comput. Ind. En. 23(10): 997-1006

Yang Miin-Shen and Yang Jenn-Hwai 2008 Machine-part cell formation in group technology using a modified ART1 method. Eur. J. Oper. Res. 188(1): 140-152

Yin Yong and Yasuda K 2005 Similarity coefficient methods applied to the cell Formation problem: a comparative investigation. Comput. Ind. Eng. 48(3): 471-489

Yin Yong and Yasuda K 2006 Similarity coefficient methods applied to the cell Formation problem: a taxonomy and review. Int. J. Prod. Econ. 101(2): 329-352

Yin Yong, Yasuda K and Hu L 2005 Formation of manufacturing cells based on material flows. Int. J. Adv. Manuf. Technol. 27: 159-165

Zolfagharia S and Liang M 2003 A new genetic algorithms for the machine/part grouping problem involving processing times and lot sizes. Comput. Ind. Eng 45(4): 713-731 